Elsevier

PAI 01104

\title{
Dimensions and components of observed chronic pain behavior
}

\author{
Johan W.S. Vlaeyen $* * * *$, Hugo Van Eek **, Nico H. Groenman *** \\ and Joop A. Schuerman $* * * * * * *$ \\ * Institute for Rehabilitation Research, ** Lucas Foundation for Rehabilitation, \\ Hoensbroek (The Netherlands), and *** Department of Medical Psychology, University of Limburg, \\ Maastricht (The Netherlands)
}

(Received 2 February 1987, revised received 26 March 1987, accepted 30 March 1987)

\begin{abstract}
Summary This study is a replication of a study done by Turk et al. [28] but under different conditions. It is an attempt to empirically examine the dimensions and components of overt and observable chronic pain behavior. A broader definition of pain behavior is chosen, namely the interaction between the pain patient and his or her direct environment. The results suggest that pain behavior can be characterized by 3 dimensions: withdrawal-approach, high arousal-low arousal and visible-audible. Furthermore, chronic pain behavior seems to be composed of at least 9 components: anxiety, attention seeking, verbal pain complaints, medication use, general verbal complaints, distorted posture and mobility, fatigue, insomnia, and depressive mood. More dimensions and components were discovered than in the study by Turk and his colleagues. However, they correspond with the variety of psychosocial problems associated with the chronic pain syndrome. This information seems to provide a useful basis for the development of an observational measurement technique for chronic pain.
\end{abstract}

Key words: Chronic pain; Pain behavior; Behavioral assessment; Multidimensional scaling

\section{Introduction}

Chronic pain is a complex phenomenon and is influenced by diverse factors. Swanson [25] introduced the conceptualization of chronic pain as a third pathologic emotion, in addition to anxiety and depression. Emotions can be characterized by a psychophysiological, a covert subjective or cognitive and an overt-motoric or instrumental factor $[9,14,22,24]$. These factors are being used more in the assessment of chronic pain $[8,17]$. Moreover, measures of each of these seem to intercorrelate

Correspondence to: J.W.S. Vlaeyen, Institute for Rehabilitation Research, Zandbergsweg 111, 6432 CC Hoenshroek, The Netherlands. 
very poorly; the amount of observed motoric behavior is often in discordance with the self-reported pain level [1.7.19.27].

A similar trichotomy is employed with regard to psychological interventions for chronic pain [29]. Interventions include respondent or physiologic methods, operant conditioning and cognitive-behavioral approaches.

In view of this 3-factor conceptualization different assessment procedures have been developed which seem to belong to 1 of the 3 factors mentioned above. For the psychophysiological factor electronic devices are used in laboratory settings. Unfortunately this kind of assessment is difficult to carry out in a clinical setting. Variables measured are electromyogram, skin resistance, heart rate and respiratory rate $[4,14]$.

The optimal way to assess the cognitive factor of pain is by the use of self-report techniques. This form of pain measurement has received most attention in the past and has been directed towards the pain experience. The best known are the McGill Pain Questionnaire [16], the visual analog scale [10], and the more recently developed Pain Perception Profile [30].

The pain diary, introduced by Fordyce [6], is probably the most frequently used assessment instrument focusing on the overt-motoric factor of chronic pain. By means of this diary, patients report about the amount of time they spend sitting, reclining and standing/walking. Research on the validity of this diary, however, gives contradictory results. Kremer et al. [12] reported negative results while Follick et al. [5] report in favor of the diary. The issue remains whether people are able to reliably describe their own instrumental behavior. The problem of self-report measures, as with all introspective methods, is that they are continuously influenced by distorting factors such as depression, anxiety or the chronicity of the problem itself [11]. Therefore observational measures, carried out by trained observers, have received increasing attention.

The observation is focused in the first place on 'pain behaviors,' a concept also introduced by Fordyce. Fordyce [6] makes a distinction between a narrow and a broader definition of pain behavior. He states that pain behavior, narrowly defined, includes:

(1) Autonomically mediated pain indicators.

(2) Visible and audible non-verhal signals that pain is experienced.

(3) Verbal reports of pain.

(4) Requests for ministrations or assistance because of pain.

(5) Functional limitation or restricted movement because of pain [6, p. 109].

In this publication, however, Fordyce remains unclear about the broader definition of pain behaviors, stating that it is not of great importance. In a more recent publication [15] we find a broader definition of pain behavior, followed by a specification:

'... pain behavior [which] is the interaction between the individual and the surrounding world. Pain behavior is defined as any and all outputs of the individual 
that a reasonable observer would characterize as suggesting pain, such as (but not limited to) posture, facial expression, verbalizing, lying down, taking medicines, seeking medical assistance, and receiving compensation [15, p. 334].'

The pain behavior construct according to the narrow definition conceptualized by Fordyce has been supported by empirical research. Turk et al. [28] employed multidimensional scaling and hierarchical clustering statistical techniques to identify the latent structure of pain behavior. They found that pain behavior can be characterized by 2 dimensions, namely audible-visible and affective-behavioral. Four clusters of pain behavior were identified and labeled: distorted ambulation or posture, negative affect, facial/audible expressions of distress, and avoidance of activity (Table I).

Although this study presents an interesting design, the question is whether the results have not been influenced by circularity. The original list of behaviors was selected from the literature, including Fordyce [6] and Sanders [20], and was thus influenced by Fordyce's ideas. After a first selection, the behaviors were sorted by pain specialists. They were members of the International Association for the Study of Pain (IASP) and were probably familiar with this conceptualization. Therefore, it is not surprising that the latent structure turns out to be generally congruent with Fordyce's original conceptualization.

Another empirical study in which the components of pain behavior are examined was done by Philips and Jahanshahi [18]. These authors presented a checklist consisting of 49 items to 267 headache patients. A factor analysis resulted in 13 factors (Table I). Based on these results the authors constructed an assessment instrument, in the form of a pain behavior checklist, giving information about the

IABLE I

EMPIRICAL EXAMINATIONS OF THE COMPONENTS OF PAIN BEHAVIOR

\begin{tabular}{lll}
\hline Authors & Turk et al. [28] & Philips and Jahanshahi [18] \\
Source & Literature & Self-report by headache patients \\
Procedure & Cluster analysis & Factor analysis \\
Components & 1. Distorted ambulation or posture & 1. Social avoidance \\
& 2. Negative affect & 2. Avoidance of housework activities \\
& 3. Facial/audible expression & 3. Daily mobility avoidance \\
of distress & 4. Activities avoidance \\
4. Avoidance of activity & 5. Daily exercise avoidance \\
& 6. Stimulation avoidance \\
& 7. Non-verbal complaint \\
& 8. Verbal complaint \\
& 9. Self-help strategies A \\
& 10. Self-help strategies B \\
& 11. Medication \\
& 12. Crying \\
& 13. Distraction \\
\hline
\end{tabular}


presence of the components of headache behavior. In this study no raters or observers were employed. The results are based on self-reported material and therefore subject to distorting factors, as discussed earlier. The possibility of transforming the checklist into an observation scale was not considered by the authors. Philips and Jahanshahi, however, conclude that the checklist has to be completed with other, more objective material.

Our study is designed to be a replication of the study of Turk et al. [28]. but with changed conditions.

(1) In contrast to Turk, our starting point is the broader definition of pain behavior, namely, the interaction between the pain patient and his direct environment.

(2) The original behavior items are not selected from the existing literature, but collected by nurses. These nurses are, at least in the clinical setting, members of the direct environment and able to observe the patient carefully.

(3) In order to avoid the problem of circularity we chose not to work with IASP members as raters, but with a more neutral group of behavioral scientists.

\section{Method}

Subjects

Information gathered by 12 nurses from the Department of Rheumatology of the Lucas Foundation for Rehabilitation who participated in an earlier study [31] was used in the first phase of the study. Ninety-six psychologists and members of the Flemish and Dutch Associations for Behavior Therapy served as subjects for the second phase of the study.

\section{Procedure}

During the first phase of the study data from an earlier study were used [31]. In this study 12 nurses were asked to carefully observe 21 male and 26 female inpatients, admitted to the Department of Rheumatology of the Lucas Foundation for Rehabilitation, for chronic back pain problems. Patients' mean ages were 39.1 and 52.0 years. Nineteen patients $(40.4 \%)$ were diagnosed as having chronic low back pain, $25.5 \%$ suffered from spondylitis ankylopoietica, $25.5 \%$ had rheumatoid arthritis and $8.5 \%$ received the diagnosis fibrositis. The systematic recording of these observations resulted in a list of 232 behavior items. For the present study only items indicating behavior which hampers the development of well-behavior were selected. Observations with great similarity were reformulated into 1 item. The final list consisted of 78 behavior items.

In the second phase, the 78 items, printed on small filing cards, were presented to the 96 psychologists with the following instruction: "You receive 78 examples of behaviors of chronic pain patients reported by nurses in a rehabilitation clinic. Please cluster these behaviors into categories based on whatever criteria that you 
believe are relevant. Do this by sorting the cards on which the behavior descriptions are printed. Behaviors that you feel belong together should be assigned to the same group. You can make as many groups as you feel are necessary.' Every psychologist produced a certain number of groups with a certain number of items. For all psychologists, the frequency of occurrence of 2 items in the same group was calculated. This information allows the construction of a $78 \times 78$ frequency matrix. Each cell represents the frequency with which 2 items coincide in the same group. These frequencies varied between 0 (pairs of items never placed together) and 96 (pairs of items always placed in the same group).

\section{Results}

The statistical techniques used were the same as in the Turk study, namely, multidimensional scaling (MDS) and hierarchical cluster analysis (HCA). MDS is a procedure aimed at the discovery of the latent structure of a data pool, when the degree of similarity between the items is known. MDS differentiates the items in various dimensions. The result is a multidimensional representation of the items in the form of a geometrical configuration of points. In this configuration, items with greater similarity are put closer to each other. Larger distances correspond to less similarity [13]. The dimensions on the basis of which the 96 psychologists sorted the 78 items were analyzed by means of the statistical MDS package Minissa, version 3.10 [2]. Solutions in 1-8 dimensions were obtained. On the basis of the respective stress values (Fig. 1) we concluded that 3 dimensions were most appropriate for the interpretation of the results.

The assignment of labels to the dimensions was based on the examination of the behavior items falling at the extreme points of each dimension.

For dimension 1, behaviors lying at one pole include 'behaves passively' and 'withdraws from other people,' while at the other pole behaviors such as 'cries' and 'requests pain medication' are found. We felt that we could assign the label 'withdrawal-approach' to this dimension.

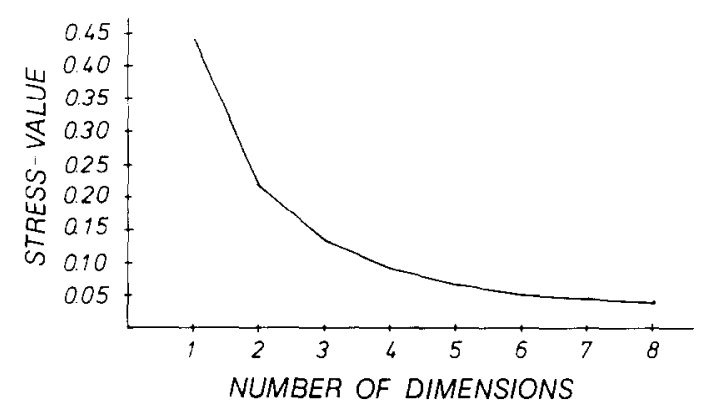

Fig. 1. MDS stress values at each of the 8 dimensions. 
TABLE II

HIERARCHICAL CLUSTER ANALYSIS OF PAIN BEHAVIORS

Items are translated from the Dutch. Letters in parentheses correspond to letters in Fig. 2.

Cluster Item

1: Anxiety

2: Attention seeking

3: Verbal pain complaints

4: Medication use

5: General verbal complaints

6: Distorted mobility and posture
Item

1. Tense (A)

15. Restless and nervous

27. Is in panic (B)

41. Nervous when having difficulties doing something

19. Mentions feeling anxious and tense

73. Blushes and sweats

16. Interrupts and disturbs others' activities

49. Talks continuously

69. Is obtrusive (C)

60. Claims the attention of nurses and other patients (D)

5. Mentions having more pain after therapy

26. Gives lively descriptions of the pain (E)

36. Says that she/he cannot stand the pain anymore

65. Complains about pain attacks

71. Complains about pain

76. Says the pain has not diminished

18. Rubs painful parts of the body (F)

75. Cries

24. Groans, moans or sighs (G)

25. Takes pain medication $(\mathrm{H})$

38. Takes more pain medication than prescribed (I)

55. Requests pain medication $(\mathrm{J})$

31. Takes sleep medication (K)

66. Takes antidepressants (L)

6. Complains about stiffness after therapy

48. Complains about stiffness after sitting (M)

61. Complains about morning stiffness

8. Asks for further specialized medical treatment

42. Asks for a consultation with the psychologist

39. Asks for help

12. Asks for a lighter therapy program

22. Says that the program is too hard $(\mathrm{N})$

35. Is querulous

32. Mentions being bored ( $O$ )

9. Needs help rising after sitting for a while

10. Needs help leaving the bed

20. Wears a brace $(P)$

37. Walks with crutches from room to the common room

45. Uses a wheelchair to go to therapy

52. Has problems with activities of daily living

17. Moves rigidly and stiffly, with stretched limbs

43. Stands in an unusual posture

33. Walks with abnormal gait

56. Has bad mobility

64. Walks guardedly and carefully 
TABLE II (continued)

\begin{tabular}{l} 
Cluster \\
\hline Item \\
63. Walks with dragging steps (Q) \\
21. Changes from one buttock to the other while sitting \\
78. Changes position frequently while sitting \\
53. Grimaces ( $R$ ) \\
58. Moves rigidly and shyly (S) \\
2. Falls asleep during the day \\
34. Lies in bed during the weekend \\
28. Lies down between therapy sessions \\
14. Takes a nap after lunch (T) \\
30. Sits between therapy sessions \\
70. Rests on the way to therapy sessions \\
72. Asks for a resting pause (U) \\
74. Sleeps between therapy sessions \\
40. Falls asleep early in the morning \\
57. Has difficulties falling asleep \\
59. Wakes up at night (V) \\
47. Awakes early in the milning (W) \\
3. Behaves passively \\
50. Does not take any initiative \\
46. Does only what has been asked \\
51. Misses therapy sessions if not reminded \\
68. Undertakes nothing outside therapy time \\
4. Is quiet and withdrawn \\
29. Isolates her/himself \\
44. Is listless \\
67. Is blue, down (X) \\
54. Worries \\
7. Talks about suicide \\
62. Talks in a pessimistic way \\
23. Says things are not working out well (Y) \\
77. Mentions not being interested when encouraged to under- \\
1ake something \\
11. Appears drowsy and sleepy \\
13. Says that she/he is tired (Z) \\
\hline
\end{tabular}

Behaviors lying at one extreme of dimension 2 include 'is obtrusive,' 'is restless and nervous' and at the other extreme we find 'walks guardedly and carefully,' 'takes a nap after lunch.' We have labeled this dimension 'high arousal-low arousal.' When comparing extreme items of this dimension with those of Turk's dimension 'affective--behavioral,' a high degree of similarity can be seen. However, we chose to label this dimension differently.

Finally, examination of the third dimension reveals that 'changes position frequently while sitting' and 'walks with abnormal gait' are found on one extreme and 'asks for lighter therapy program,' 'mentions having more pain after therapy' on the other. This dimension shows much similarity with the dimension 


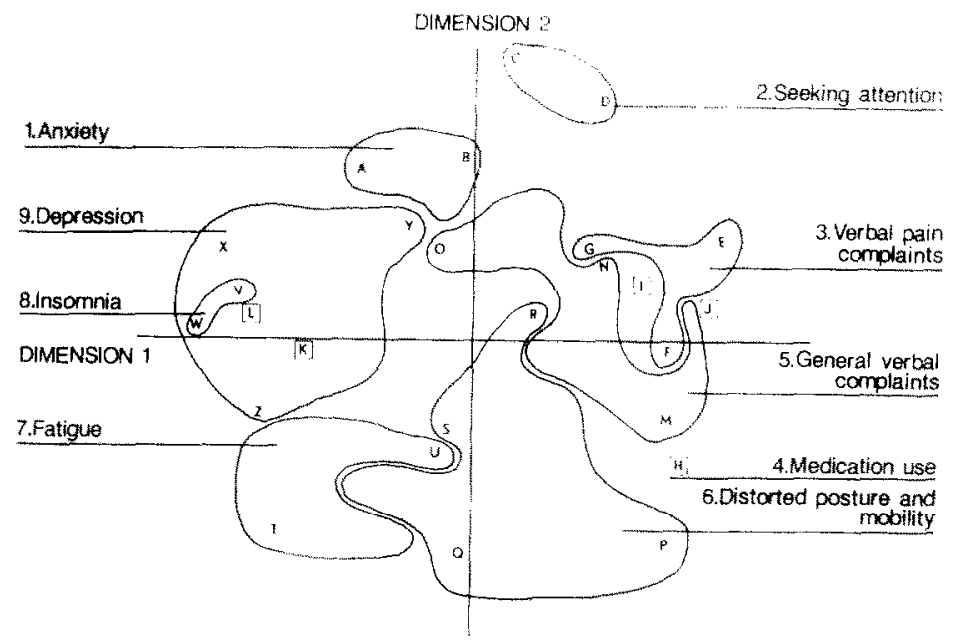

Fig. 2. Graphic MDS 2-dimensional representation with clusters embedded.

'visible-audible' in the study of Turk and his colleagues. Therefore we gave it the same label.

The next step consisted of looking at the different behavior components. The original similarity matrix was transformed into a distance matrix and subsequently submitted to HCA. HCA is aimed at grouping items with a relatively high degree of similarity [3], in contrast to MDS which helps to differentiate items.

HCA resulted in 9 different clusters of behaviors. Fig. 2 shows these clusters embedded in the geographical MDS representation. Based on inspection of the items of each cluster, listed in Table II, the clusters were labeled as: (1) anxiety; (2) attention seeking; (3) verbal pain complaints; (4) medication use; (5) general verbal complaints; (6) distorted mobility and posture; (7) fatigue; (8) insomnia; and (9) depression.

\section{Discussion}

Chronic pain behavior, considered as the overt-motoric factor of the chronic pain syndrome and defined as the interaction between the chronic pain patient and his/her direct environment, seems to be composed of 9 components and to be characterized by 3 dimensions.

The dimensions and components found in our study appear to differ slightly from those found by Turk and his colleagues [28]. We opted for a broader definition of pain behavior than Turk, and found one more dimension and about twice as many components. The results of our study suggest that chronic pain patients present a behavior pattern which is more extensive than the typical pain behaviors found in the literature. Anxiety, depression and insomnia are not only reported in 
relation to chronic pain [21,23], these complaints have been empirically confirmed in our study.

The differences with the study of Philips and Jahanshahi [18] are more significant. Only 4 of their components (medication use, verbal complaints, non-verbal complaints and avoidance of mobility) are confirmed in our study. One of the reasons might be the different conditions of the experiment. Philips and Jahanshahi used self-reported behavior items including items indicating well-behavior. Of interest is that their results, compared to ours, suggest that headache patients display another pain behavior pattern than our chronic pain patient population.

The results not only give insight into the latent structure of the 'broader' pain behavior construct as introduced by Fordyce [6,15], they also provide a basis for the construction of an observation scale for the instrumental factor of chronic pain, or overt pain behavior. The validity of the clusters found could be assessed by means of a checklist in which the 78 items are provided with a rating scale. This checklist could then be used by nurses or trained observers. Moreover, such a checklist would provide the possibility of comparing pain patients with different diagnoses, and thus contribute to the assessment of chronic pain in general.

For each patient an observation profile could be formed, which could provide information about at least 2 aspects of the observed chronic pain behavior. First, the position of the patients' pain behavior in relation to the 3 dimensions can be calculated, as MDS indicates a loading for each item with respect to each of the 3 dimensions. Second, as every item belongs to 1 of the 9 clusters, a score for each cluster can also be calculated, based on the ratings given to the items of the respective clusters.

Assessment should be related to treatment. Although answers are premature at this moment, the question arises whether on the basis of the profile proposed above conclusions could be drawn about the therapeutic intervention to be chosen. It is not impossible that patients scoring higher on the dimension 'high arousal' can benefit more from respondent techniques such as relaxation. High scorers in the direction of 'approach' are probably sensitive to an operant approach, aimed at abolishing excessive behavior and shaping more adequate well-behavior. Finally, pain behavior merely characterized by 'withdrawal' might be more controlled by cognitive factors or the pain experience. In this case cognitive-behavioral interventions might be more appropriate.

More clinical evaluation research, including research towards the predictive value of specific assessment procedures, is essential in order to test these tentatively formulated hypotheses.

Although the present study should improve the assessment of pain, it only focused on one aspect of the chronic pain syndrome. A complete assessment should also include an examination of the cognitive and the psychophysiological factors. The behavioral analysis which includes such a multifactorial assessment is considered to be of great importance in the diagnosis and treatment of chronic pain. Wc agree with Syrjala and Chapman [26] that a pain profile, similar to the Minnesota Multiphasic Personality Inventory (MMPI), would be ideal. Such a profile should be based upon observational, self-report and psychophysiological data. 


\section{Acknowledgements}

The authors express their gratitude to A. Bakker-Boerrigter. M.D., and the staff of the Department of Rheumatology of the Lucas Foundation for Rehabilitation at Hoensbroek, The Netherlands, and the members of the Flemish and Dutch Associations of Behavior Therapy. They also wish to thank Prof. J.J.C.B. Bremer, Ph.D., and Ms A.M.J. Snijders for critically reviewing the manuscript, Mr. J. van Houtem for his exact statistical advice and Mrs. T. Fasott-Dumont for the data processing.

\section{References}

1 Cosyns, P. et Vlaeyen, J., La douleur chronique. In: O. Fontaine, J. Cottraux et R. Ladouceur (Eds.). Cliniques de Thérapie Comportementale, Mardaga, Liège, 1983.

2 Davies, P.M. and Coxon. A.P.M. MDS(X) User Manual. The MDS(X) Series of Multidimensional Scaling Programming, Inter-University Research Council Series, Report no. 55. University of Edinburgh, 1983 .

3 Everitt, B., Cluster Analysis, Heinemann, London, 1974.

4 Flor, H., Turk, D.C. and Birbaumer, N., Assessment of stress-related psychophysiological reactions in chronic back pain patients, J. consult. clin. Psychol.. 53 (1985) 354-364.

5 Follick. M.J., Ahern, D.K. and Laser-Wolston. N.. Evaluation of a daily activity diary for chronic pain patients. Pain, 19 (1984) 373-382.

6 Fordyce, W.E., Bchavioral Mcthods for Chronic Pain and Illness, Mosby, St. Louis, MO, 1976.

7 Fordyce, W.E., Lansky, D., Calsyn, D.A., Shelton. J.L.. Stolov, W.C. and Rock. D.L.. Pain measurement and pain behavior, Pain, 18 (1984) 53-69.

8 Fredericksen, L., Sterling. R. and Ross, J., Methodology in the measurement of pain. Behav. Ther. 9 (1978) $486-488$.

9 Hugdahl, K., The three-systems-model of fear and emotion, a critical examination, Behav. Res. Ther.. 19 (1981) 75-85

10 Huskisson, E.C., Visual analog scales. In: R. Melzack (Ed.), Pain Measurement and Assessment, Raven Press. New York, 1983.

11 Kremer, E.F.. Block. A. and Atkinson, J.H. Assessment of pain behavior: factors that distort self report, In: R. Melzack (Ed.), Pain Measurement and Assessment, Raven Press, New York, 1983.

12 Kremer, E.F., Block. A. and Gaylor, M.S., Behavioral approaches to treatment of chronic pain: the inaccuracy of patient self-report measures, Arch. phys. Med. Rehab., 62 (1981) $188-191$.

13 Kruskal, J.B. and Wish, M., Multidimensional Scaling, Sage, Beverly Hills, CA, 1978.

14 Lang. P.J. Fear reduction and fear behavior: problems in treating a construct. Res. Psychother.. 3 (1968) 90-102.

15 Loeser, J.D. and Fordyce. W.E., Chronic pain. In: J.E. Carr and H.A. Dengerink (Eds.), Behavioral Science in the Practice of Medicine, Elsevier, Amsterdam, 1983.

16 Melzack, R., The McGill Pain Questionnaire, major properties and scoring methods, Pain. 1 (1975) $277 \ldots 299$.

17 Philips, C.. Assessment of chronic headache behavior. In: R. Melzack (Ed.). Pain Measurement and Assessment, Raven Press. New York, 1983.

18 Philips, C.. and Jahanshahi, M., Validating a new technique for the assessment of pain behavior, Behav. Res. Ther., 24 (1986) 35-42.

19 Richards, J.S., Nepomuceno, C., Riles, M. and Suer, Z.. Assessing pain behavior: the UAB pain behavior scale, Pain, 12 (1982) 393-398.

20 Sanders, S.H., Toward a practical instrument for the automatic measurement of 'up-time' in chronic pain patients, Pain, 9 (1980) $103-109$.

21 Savitz, D., Medical evaluation of the chronic pain patient, In: G.M. Aronoff (Ed.), Evaluation and Treatment of Chronic Pain, Urban and Schwarzenberg. Baltimore, MD, 1985. 
22 Schachter, S. and Singer, J.E., Cognitive, social and physiological determinants of emotional state, Psychol. Rev., 69 (1962) 379-399.

23 Sternbach, R.A., Pain patients: Traits and Treatment, Academic Press, New York, 1974.

24 Strongman, K.T., The Psychology of Emotion (2nd Edn.), Wiley, New York, 1979.

25 Swanson, D.W., Chronic pain as a third pathologic emotion, Amer. J. Psychiat., 141 (1984) 210-214.

26 Syrjala, K.L. and Chapman, C.R., Measurement of clinical pain: a review and integration of research findings. In: C. Benedetti et al. (Eds.), Advances in Pain Research and Therapy, Vol. 7, Raven Press, New York, 1984.

27 Teske, K., Daut, R.L. and Cleeland, C.S., Relationships between nurses' observations and paticnts' self-reports of pain, Pain, 16 (1983) 289-296.

28 Turk, D.C., Wack, J.T. and Kerns, R.D., An empirical examination of the 'pain-behavior' construct, J. behav. Med., 8 (1985) 119-130.

29 Turner, J.A. and Romano, J.A., Evaluating psychologic interventions for chronic pain: issues and recent developments. In: C. Benedetti et al. (Eds.), Advances in Pain Research and Therapy, Raven Press, New York, 1984.

30 Tursky, B., Jamner, L.D. and Friedman, R., The Pain Perception Profile: a psychophysical approach to the assessment of pain report, Behav. Ther., 13 (1982) 376-394.

31 Vlaeyen, J.W.S., Van Eek, H., Groenman, N.H. and Schuerman, J.A., Construction of an observation scale for the behavior of pain patients, Paper presented at the 2nd Europ. Conf. on Research in Rehabilitation, Düsseldorf, 18-19 Nov. 1985. 